\title{
Aggressive Periodontitis with Streptococcal Gingivitis: A Case Report
}

\author{
Cankat Karaa, DDS, PhD \\ Turgut Demira, DDS, PhD \\ Adnan Tezela, DDS, PhD \\ Meltem Zihnia, DDS
}

\begin{abstract}
Acute streptococcal gingivitis is an acute inflammation of the oral mucosa and also may be seen with the other oral diseases as aggressive periodontitis that is characterized by a considerable attachment loss over a relatively short period of time. Streptococcal infections of gingiva are seen rarely; also the origin of this gingival inflammation is occasionally different from that of routine plaque-associated gingivitis. The clinical features and treatment methods of these diseases are already reported in previous literatures. This case report describes a patient who presented with severe gingival inflammation and attachment loss that was diagnosed as an acute streptococcal infection associated with aggressive periodontitis. In this study a supportive treatment option was demonstrated based on these data and antacid treatment as adjunctive to the recommended treatment modalities was used for streptococcal gingivitis. (Eur J Dent 2007;1:251-255)
\end{abstract}

Key words: Acute streptococcal gingivitis; Aggressive periodontitis; Gingival inflammation; Attachment loss.

\section{INTRODUCTION}

Acute streptococcal gingivitis is an acute inflammation of the oral mucosa and the pathogens implicated in gingival inflammation is rarely different from that of routine plaque-associated gingivitis such as Neisseria gonorrhea, Treponema pallidum and recently streptococci infections. ${ }^{1,2}$ Streptococci, which can be seen in the composition of microbial dental plaque, may cause inflammatory periodontal disease and dental caries. ${ }^{3}$ During the International Workshop for a Classification

1. a Department of Periodontology, Faculty of Dentistry, Atatürk University, Erzurum, Turkey

- Corresponding Author: Cankat KARA Atatürk University, Faculty of Dentistry Department of Periodontology, Erzurum/TURKEY E-mail: mcankatahotmail.com of Periodontal Disease and Conditions in 1999, a new classification of disease was introduced and American Periodontology Academy classified the streptococcal periodontal disease under the group of gingival disease due to this specific bacterium has poor related with microbial plaque formation. Although the streptococci related disease of gingiva has very specific microbiological and clinic features, there have been only a few cases reported in the literature. Another significant feature of streptococcal gingivitis is that, it can be seen on gingiva with the other inflammatory diseases like throat inflammations. ${ }^{4.5}$ However, the previous data about the combination of streptococcal gingivitis and other periodontal diseases as aggressive periodontitis (AP) is unclear.

The purpose of this case report was two-fold; 
first, to show how streptococcal infections can be effective to cause severe periodontal damage in AP and second, to test a supportive treatment modality which may be very effective to this devastating condition.

\section{CASE REPORT}

The patient, a 26-year-old female, was referred to our clinic by her general dentist for severe bleeding gums, gingival hyperplasia, and halitosis. At the time, the patient was experiencing pain, severe bleeding with brushing related to her periodontal disease. She had a non-contributory medical history. However, prior to her visit to our clinic, she had never had a periodontal examination. On examination, generalized edematous, hyperplastic gingiva, bleeding on probing and calculus formation was seen (Figure 1). To determine the clinical situation of the subject, gingival index, ${ }^{6}$ plaque index, ${ }^{7}$ periodontal probing depths (PPD) and clinical attachment levels (CAL) of the teeth were recorded before and after the treatment by using a manual probe ( $\mathrm{Hu}$ Friedy Manufacturing Inc., Chicago, IL, USA) (Table

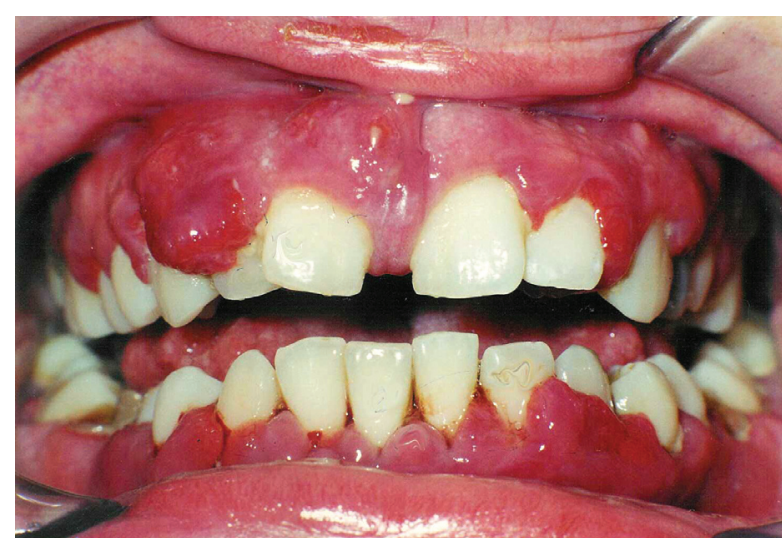

Figure 1. Pre-treatment clinical view of anterior region of the oral cavity showing severe gingival inflammation diffused periodontal and gingival abscess formation.
1). Periodontal examination revealed gingival swelling, $78.2 \%$ bleeding at probing sites, $52.7 \%$ plaque control record, and $72.9 \%$ of the sites had a periodontal pocket depth of $4 \mathrm{~mm}$ or more. Upon initial examination, radiographs demonstrated extensive generalized vertical intra-bony defects in the maxillary and mandibular arches (Figure 2). Based on the clinical and radiographic findings, a diagnosis of generalize AP was assigned to the patient. According to these findings, treatment was started with an initial phase of mechanical therapy; including systematic scaling and planing of all accessible root surfaces and the introduction of meticulous oral hygiene. During oral hygiene instruction and subsequent initial preparation as a chemotherapeutic support tetracycline lonly first day $2 \times 100 \mathrm{mg}$ and $1 \times 100 \mathrm{mg} /$ day - Tetradox, Fako, Turkeyl had been given to the patient with a mouth rinse including Chlorhexidine Digluconate $(0.12 \%)$ and Benzidamin HCL $10.15 \%$ ) (Kloroben, Drogsan, Turkey). And also to improve the patient's oral and dental hygiene, a new soft toothbrush was referred for use as part of the oral care protocol. After optimal oral hygiene was provided, based on persistence of periodontal lesions, a second phase of therapy was planned and the advanced periodontal therapy, flap and gingivectomy operations were done and remaining roots were

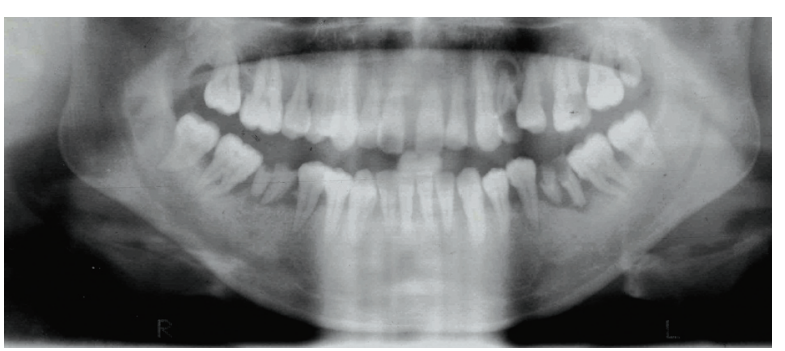

Figure 2. Panoramic radiograph showing generalize bone loss; especially at the molar areas.

Table 1. Clinical parameters of patient assessed at baseline (TO), after initial treatment (T1), after periodontal surgery (T2) and after the all periodontal treatments (T3).

\begin{tabular}{lccccc}
\hline & T0 & T1 & T2 & T3 \\
\hline PI & 2.34 & 0.67 & 0.51 & 0.18 \\
GI & 2.81 & 0.95 & 0.35 & 0.05 \\
PPD & 6.41 & 5.13 & 4.09 & 4.01 \\
CAL & 4.96 & 4.85 & 4.05 & 3.91 \\
\hline
\end{tabular}

PI, Plaque Index; GI, Gingival Index; PPD, Periodontal Probing Depths; CAL, Clinical Attachment Levels. 
removed. After resolution of the periodontal infection (a period of seven weeks), the patient was placed on an individually tailored maintenance care program including continuous evaluation of the occurrence and the risk of disease progression. However, after a week, bleeding, edema and dark red discoloration on the marginal areas of gingiva developed again (Figure 3). Further medical tests were carried out to look for searching an underlying systemic disease and its impact on the etiology of the disease. Because of the results were in normal limits, we suspected acute streptococcal infection. Although acute streptococcal infection was suspected because of the clinical features of the patient, for being sure bacterial sample was obtained by scraping the surfaces of the gingival lesions with a sterile chip and the anaerobic-culture technique were used to identify the bacteria. Cultures of the gingival samples grew streptococcus pyogenes and a few other microorganisms. To prevent antibiotic resistance development with chemotherapeutic agents in the treatment of aggressive periodontitis, we decided to use an alternative treatment primarily, which was based on the microorganism's growth characteristics. The acidic oral $\mathrm{pH}$ required for growing of the Streptococcus species was tried to change and the optimal oral $\mathrm{pH}$ in normal values was kept. For this purpose, an antacid chewing tablet (Rennie 680mg, Roche, Turkey), which includes calcium and magnesium carbonate, was offered two times a day for a week. To determine the effectiveness of this medication, normal whole saliva $\mathrm{pH}$ and whole saliva $\mathrm{pH}$ after using the

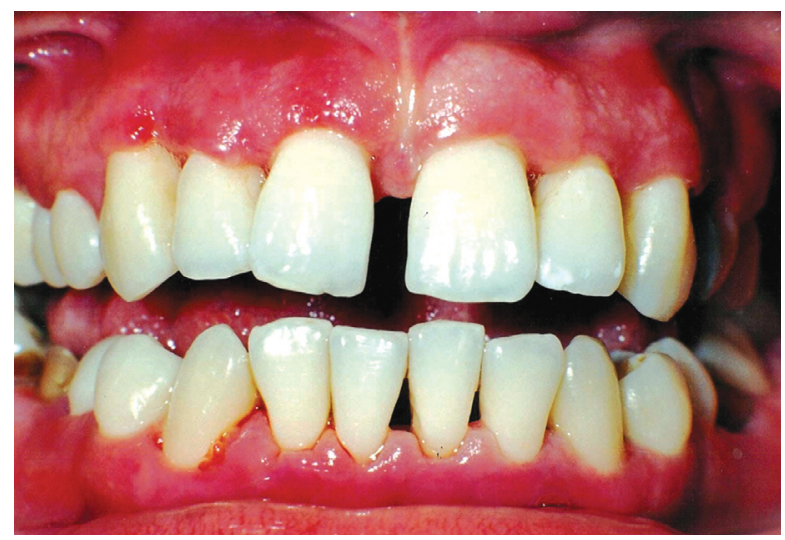

Figure 3. Clinical view of anterior region of the oral cavity after the treatment for aggressive periodontitis showing acute streptococcal gingivitis. antacid were measured (Inolab pH meter level 2, Wissenschafllich Technische, Germany) (Figure 4). This normal value was picked up of the same patient in different moments. At the end of this additional treatment, oral health was obtained (Figure 5).

\section{DISCUSSION}

In a revised classification system for periodontal diseases, AP was redefined to comprise a complex entity of microbial alterations and cellular dysfunctions that differentiate the underlying molecular mechanisms from chronic periodontal disease. ${ }^{8}$ The aggressive nature of this disease process is due in part to A. actinomycetemcomitans, which is regarded as the key etiological agent based on studies reporting a frequency rate of nearly $90 \%$ in periodontal lesions. ${ }^{9}$ There is a consensus about the method and application of the treatment for AP. Once AP has been diagnosed, a comprehensive periodontal treatment plan must be developed. The treatment of periodontal diseases is divided into four phases: systemic, hygienic, corrective and maintenance / supportive therapy. As treatment progresses through the four phases, the dentist uses both surgical and non-surgical therapy to remove biofilm created by the bacterial pathogens; this procedure is in agreement with good medical practice because the bacterial load should be reduced as much as possible prior to the use of antibiotics. The adjunctive systemic use of antimicrobials along

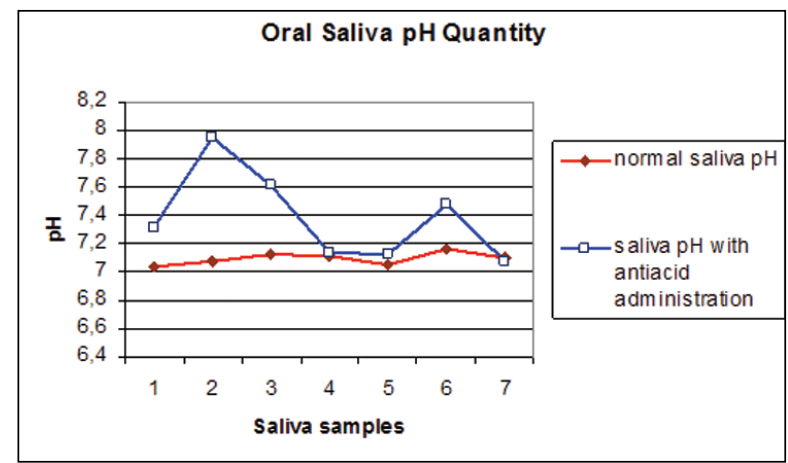

$1^{\text {st }}$ sample: Without any intervention.

$2^{\text {nd }}$ sample: 5 minutes after oral antiacid administration.

$3^{\text {rd }}$ sample: 30 minutes after oral antiacid administration. $4^{\text {th }}$ sample: 60 minutes after oral antiacid administration. $5^{\text {th }}$ sample: 6 hours after oral antiacid administration. $6^{\text {th }}$ sample: 6.5 hours after oral antiacid administration. $7^{\text {th }}$ sample: After using the antacid two times a day for one week.

Figure 4. PH values of normal and with antacid administration saliva samples. 
with mechanical debridement of the root surfaces to disrupt the biofilm is effective in most patients with AP. ${ }^{10}$

Tetracycline that was used by most of the researchers in previous studies was chosen for chemotherapy because of its affectivity for periodontal diseases and high secretion capacity from gingival tissue. ${ }^{11}$ The patient was also treated according to these recommendations. Although a successful and appropriate treatment was applied to the patient according to the recommendations for AP, streptococcal inflammation increased the worsening of the oral findings present in AP.

Acute streptococcal gingivitis has been given little attention in the dental literature in general, and in the periodontal literature in particular. Only one well-documented study is cited in the medicodental literature. ${ }^{4}$ The treatment of choice for streptococci infections is penicillin. Sulfonamides and broad spectrum antibiotics have also been used to treat streptococcal infections. Recognition, identification of etiology, diagnosis of disease, and treatment of acute gingivostomatitis are critically important of untreated group A beta-hemolytic streptococcal infection, which has many serious complications. ${ }^{12}$

In addition to this primary etiologic factor, other factors such as differentiation of saliva composition can foster progression of this disease. In progression of this disease like other periodontal diseases, saliva plays important roles as a disease marker and as a defense mechanism.

Saliva has some antimicrobial activity against many different microorganisms. This is mainly

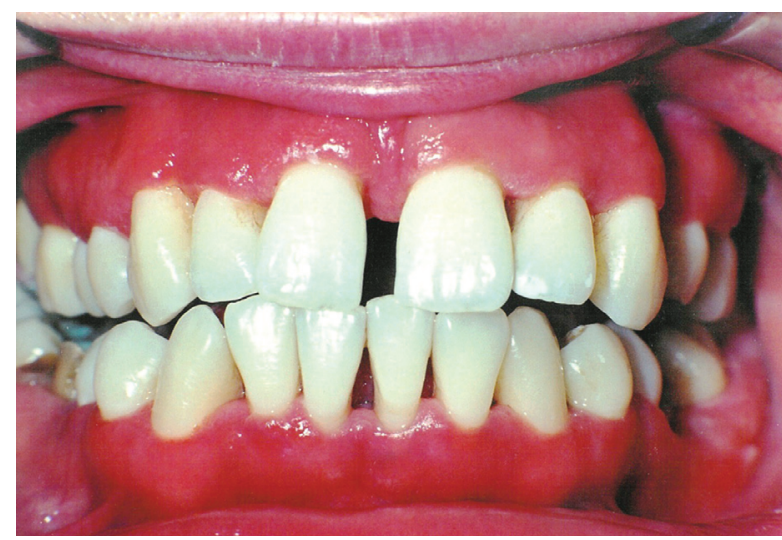

Figure 5. Post-treatment clinical view of anterior region of the oral cavity; observe reduction of gingival inflammation and acceptable gingival health. due to the presence of immunoglobulin and nonimmunoglobulin agents in its content. ${ }^{13}$ It also prevents the proteins and cells in oral mucosa from $\mathrm{H}_{2} \mathrm{O}_{2}$ toxicity. ${ }^{14}$ At physiologic concentrations and neutral $\mathrm{pH}$, it prevents the bacterial glycolysis by inhibiting the $\mathrm{pH}$ and potentiates the antibacterial defense mechanisms as a bacteriostatic agent. ${ }^{15,16}$ It has been shown that the OHSCN/ OSCN value had a stronger anti-streptococcal effect and inhibited the bacterial growth very effectively if it was sufficiently present enough in the saliva in $\mathrm{pH}$ values of $7 .{ }^{17}$ The $\mathrm{pH}$ of saliva increases with concomitant secretion of $\mathrm{HCO}_{3}$ with saliva secretion (5.5-7.5). The most important factor for the increase of the $\mathrm{pH}$ is the $\mathrm{HCO}_{3} \cdot{ }^{18}$ Even though saliva has all those beneficiary antimicrobial effects that were mentioned above, sometimes it may not be sufficient enough to kill some specific bacteria which can be available in oral $\mathrm{pH}$ values of 6-8 and for streptococcus species which can survive at a low $\mathrm{pH}$ and to continue producing acid. In conclusion, using an antacid agent may prove to be useful as an indicator of environmental conditions in the oral cavity, and as a determinant of treatment model among oral streptococci.

\section{CONCLUSIONS}

With this case report an alternative treatment option based on these data was demonstrated and antacid treatmentasadjunctivetotherecommended treatment modalities for streptococcus gingivitis was used. It can be said that oral antacid treatment as well as conventional periodontal treatment may be helpful in the treatment of oral infections due to Streptococcus.

\section{REFERENCES}

1. Holmstrup P. Non-plaque-induced gingival lesions. Ann Periodontol 1999;4:20-29.

2. Pindborg JJ. Atlas of disease of the oral mucosa. 3th ed. Copenhagen: Munksgaard; 1980:24-25.

3. Lindhe J. Textbook of Clinical Periodontology. 2nd ed. Copenhagen: Munksgaard; 1992:92-128.

4. Littner MM, Dayan D, Kaffe I, Begleiter A, Gorsky M, Moskana D, Buchner A. Acute streptococcal gingivostomatitis. Report of five cases and review of the literature. Oral Surg Oral Med 1982;53:144-147.

5. Carranza FA. Glickman's Clinical Periodontology. 7th ed. Philadelphia: W.B. Saunders Company; 1990:149-165. 
6. Löe H, Silness J. Periodontal diseases in pregnancy. Part I. Correlation and severity. Acta Odontologia Scandinavian 1963;21:533-551.

7. Silness $J$ and Löe H. Periodontal diseases in pregnancy. Part II. Correlation between oral hygiene and periodontal control. Acta Odontologia Scandinavian 1964;22:121-135.

8. Armitage GC. Development of a Classification System for Periodontal Diseases and Conditions. Ann Periodontol 1999;4:1-6.

9. Nonnenmacher C, Mutters R, Flores de Jacoby L. Microbiological characteristics of subgingival microbiota in adult periodontitis, localized juvenile periodontitis and rapidly progressive periodontitis subjects. Clin Microbiol Infect 2001;7:213-217.

10. Dörfer CE. Antimicrobials for the treatment of aggressive periodontitis. Oral Diseases 2003;9(s1):51-53.

11. Mandell RL, Socransky SS. Microbiological and clinical effects of surgery plus doxycycline on juvenile periodontitis. J Periodontol 1988;59:373-379.

12. Katz J, Guelmann M, Rudolph M, Ruskin J. Acute streptococcal infection of the gingiva, lower lip, and pharynx-A case report. J Periodontol 2002;73:1392-1395.

13. Tenovuo J, Valtakoski J. The correlation between salivary peroxidase activity salivary flows 1.FDI.Saliva: Its role in health and disease. Int Dent $J$ 1992;42:291-304.

14. Tenovuo J, Larjava $H$. The protective effect of peroxidase and thiocyanate against hydrogen peroxide toxicity assessed by the uptake of $(3 \mathrm{H})$-Thymidine by human gingival fibroblasts culture in vitro. Arch Oral Biol 1984;29:445-451.

15. Kersten HW, Moorer WR, Wewer R. Thiocyonate as a cofactor in myeloperoxidase activity against sreptococcus mutans. J Dent Res 1981;60:831-837.

16. Rahemtulla MB, Baldone DC, Pruitt KM, Rahemtulla F. Effect of variations in $\mathrm{pH}$ and hypothiocyanite concentrations on S. mutans glucose metabolism. J Dent Res 1987;66:486491.

17. Lumikoni M, Soukka T, Nurmio S, Tenovuo J. İnhibition of the growth of streptoccus mutans, streptococcus sabrinus and lactobacillus casei by oral peroxidase systems in human saliva. Arch Oral Biol 1991;3:155-160.

18. Güven $\mathrm{Y}$. The importance of $\mathrm{pH}$ and buffering in dentistry. Tübitak Oral Biology and Practical Education Programme $1981 ; 1-6$. 